\title{
The rhodium, silver and indium content of some chondritic meteorites
}

\author{
U. SCHINDEWOLF* and M. WAHLGREN \\ Department of Chemistry, University of Michigan, Ann Arbor, Michigan
}

(Received 23 April 1959)

\begin{abstract}
By neutron activation analysis the rhodium, silver and indium content of five chondritic meteorites has been determined. The average data are 0.186 and 0.094 p.p.m. for rhodium and silver. The upper limit for the indium content is 0.001 p.p.m. The values for rhodium and silver are in fair agreoment with the data estimated by SuEsS and UREY; the indium values, however, are lower by a
\end{abstract} factor of 100 or more.

\section{INTRODUCTION}

KNOWLEDGE of the abundance distribution of nuclear species on a cosmic scale is a prerequisite to the formulation of any theory of the formation of the elements. SUESS and UREY (1956) in a critical discussion of available abundance data taken mainly from analyses of stony meteorites have reported the apparent unreliability of previous analytical data for a number of trace elements. By activation analysis new abundance data for five chondritic meteorites have been obtained in this laboratory for three of these elements (rhodium, silver and indium), utilizing their neutron capture products $4.4 \mathrm{~min} \mathrm{Rh}^{104}, 2 \cdot 3 \mathrm{~min} \mathrm{Ag}^{108}$ and $54 \mathrm{~min} \mathrm{In}^{116 m}$. The short half-lives and favourable cross-section for the formation of these nuclides permit the use of relatively short irradiations but require rapid and simple chemical separations. Use was made of the Ford Nuclear Reactor of the Phoenix Memorial Laboratory with its pneumatic tube system (MEINke, 1959) which allowed short and precisely timed irradiations and fast delivery of the samples after the irradiation.

A fusion technique was developed to achieve rapid dissolution of the finely powdered meteoritic samples and the existing radiochemical separation methods for the three elements of interest were modified to allow their fast chemical separation. The flux available for these studies was $10^{11}$ neutrons $/ \mathrm{cm}^{-2} / \mathrm{sec}^{-1}$ at a power level of $0.1 \mathrm{MW}$. On the basis of some earlier estimations (SCHINDEWOLF, 1958) it was expected that with this neutron flux and irradiation times of one half-life of the nuclides of interest a sensitivity of $10^{-8} \mathrm{~g}$ for rhodium and for silver and $10^{-9} \mathrm{~g}$ for indium could be obtained allowing for a separation time of two half-lives. 'This seemed to be sufficient because corresponding to the calculations of SUESS and UREY the abundance of these elements should be in the order of $10^{-7} \mathrm{~g} / \mathrm{g}$ of meteorite.

\section{EXPERIMENTAL}

\section{Preparation of the meteoritic material}

After etching the surface with $\mathrm{HCl}$ and rinsing it with distilled water, the meteorites were ground to a very fine powder with a steel mortar and a vibrating

* Present address: Institut für Kernuerfahrenstechnik der Technischen Hochschule, Karlsruhe, Germany. 
electric ball mill.* Samples of $0 \cdot 5-1 \cdot 0 \mathrm{~g}$ were weighed out and transferred to a small lusteroid capsule which was placed inside a rapid opening pull-cap nylon "rabbit" for irradiation. Its design was such that the samples could be removed and the chemical separation started within a few seconds after the end of the irradiation.

\section{Dissolution of the meteoritic material}

The samples were dissolved in about $8 \mathrm{~g}$ of molten sodium peroxide in a nickel crucible which was brought to a dull red heat over a Meker burner just before the end of the irradiation. Care had to be taken not to decompose the peroxide by heating too long or too vigorously before adding the meteorite powder, for otherwise it might not dissolve the material completely. The fusion was allowed to proceed for $1-1 \frac{1}{2} \mathrm{~min}$, then the outside of the crucible was cooled by dipping it in a beaker of cold water, and the melt was made to solidify in a thin, readily dissolved coating by manipulation of the crucible. The melt was then dissolved by immersion into an aqueous solution (about $100 \mathrm{ml}$ ) containing sufficient $\mathrm{HCl}$ to neutralize all the sodium peroxide and leave a slightly acid solution.

\section{Chemical separations}

(a) Rhodium. About $20 \mathrm{mg}$ of rhodium carrier were added to the solution before adding the solidified melt. After its quick dissolution saturated sodium nitrite solution was added to the boiling solution until it was slightly basic. Rhodium is complexed by the nitrite ions whereas all iron and also silica, the main constituents of the stony meteorites, are precipitated. The iron so removed acts as a scavenger for many trace elements. After fast filtration in a large Buchner glass fritted funnel a few grammes of potassium nitrite were added to the solution and the precipitated potassium-rhodium-nitrite complex filtered off and washed. It then was dissolved in hot 6-8 $\mathrm{N} \mathrm{HCl}$, the rhodium reduced to the metallic state by adding zinc powder, filtered in a filter chimney, washed with dilute acid, water and alcohol, and finally mounted on a counting card. The determination of the chemical yield was done after the counting by weighing the metallic rhodium. From the end of the bombardment until the beginning of the counting not more than $9 \mathrm{~min}$ were required. The isotopic exchange between the rhodium carrier and the activated rhodium traces of the meteorite was confirmed with 220 day $\mathrm{Rh}^{102}$. The separation factors for the elements tested (one representative for each group of the periodic table, Cs, Sr, Ce, $\mathrm{Zr}, \mathrm{Nb}, \mathrm{W}, \mathrm{Tc}, \mathrm{Ru}, \mathrm{Ag}, \mathrm{Zn}, \mathrm{In}, \mathrm{Sn}, \mathrm{Sb}$, $\mathrm{Se}, \mathrm{Re}$ ) werc greater than $10^{5}$.

(b) Silver. The silver carrier was added to the nickel crucible and dried before addition of the sodium peroxide and the preliminary fusion. After fusion the melt was dissolved in $\mathrm{HCl}$, the resulting silver chloride was filtered off, washed and dissolved in hot ammonia. A few milligrams of iron-III were added as scavenger. Finally, the silver was reduced with zinc powder, filtered in a filter chimney, washed with dilute acid, water and alcohol, and mounted on a counting card. The chemical yield was determined after counting by titration with thiocyanate. * High speed vibration mill with agate sample tube and balls, available from Jarrell Ash Co.,
Newtonville, Mass. 
The elapsed time between the end of the bombardment and the beginning of the counting was about $5 \mathrm{~min}$. The completeness of the chemical exchange of added carrier and the activated traces was checked with 210-day $\mathrm{Ag}^{110}$. The separation factors from other elements were not determined, however, the procedure described here has been proven to be very useful for the effective and clean separation of short-lived silver isotopes from fission products (ALwXANDER et al., 1958).

(c) Indium. After dissolving the cooled melt in water containing $20 \mathrm{mg}$ of indium carrier, an equal amount of $9 \mathrm{~N} \mathrm{HBr}$ was added and the solution heated to boiling. In $\mathrm{Br}_{3}$ was extracted twice with $40 \mathrm{ml}$ portions of ether and the combined portions were washed twice with $20 \mathrm{ml}$ of $4.5 \mathrm{~N} \mathrm{HBr}$. The indium was then stripped from the ether phase with $30 \mathrm{ml}$ of $6 \mathrm{~N} \mathrm{HCl}$. The solution was boiled down almost to dryness, taken up in $2 \mathrm{~N} \mathrm{HCl}$ and passed through an anion exchange column (Dowex 1,8 per cent cross-linking, 100 to 200 mesh). This step very effectively separates indium from iron which goes with the indium into the ether but is absorbed very strongly on the column. The eluted indium solution is scavenged with a palladium sulphide precipitation. After buffering the solution with acetate the indium was precipitated as the sulphide, filtered off and airdried. When necessary the scavenging step and indium sulphide precipitation were repeated. The sulphide precipitate was weighed for the chemical yield determination. The procedure takes about $1 \mathrm{hr}$. The separation factors from other elements have not been determined, however, they are estimated to be in the order of $10^{5}$ (SUNDERMAN et al., 1959).

\section{Preparation of the standards}

The standards were prepared by pipeting a suitable aliquot of the standard solution on to a small square of tissue paper dried under a heat lamp and carefully wrapped in two more layers of tissue paper. Owing to the short half-lives of the isotopes of interest the standards and the unknowns could not be irradiated simultaneously. Therefore each one was combined with a weighed gold foil ( $1 \mathrm{mg}$ ), in which the induced activity of $2 \cdot 8$ day $\mathrm{Au}^{198}$ served as a neutron monitor. For the experiments described here no neutron flux variations could be detected over the small errors of the foil weighings and the counting statistics.

\section{Counting techniques}

The dried samples on filter paper were centred on a cardboard counting card and covered with cellophane. The samples were counted in a side window flowtype $\beta$-proportional counter and the decay followed to a constant background. This required about 20-30 min for the rhodium and silver samples; for indium sce below.

\section{Reproducibility of the procedure}

The validity of the analysis was confirmed for each isotope by adding a known amount of the standard to the meteorite sample and carrying through the complete irradiation and separation. When from 5 to $10 \mu \mathrm{g}$ of the elements of interest were added to $1 \mathrm{~g}$ of meteoritic material the results were accurate to within 10 to $15 \%$. The precision of the data in pure meteoritic samples will be much lower 
because of the smaller abundance of these elements. The relatively good agreement between the amount of rhodium, silver or indium added to the meteorites and the ones found by activation analysis confirms that there is no appreciable absorption or slowing down of neutrons in the meteorite or the added gold foil which might change the neutron flux. Owing to the difficulties of working with short-lived nuclides a better agreement can hardly be expected.

\section{Results and Discussion}

The abundance data (in p.p.m.) obtained in the experiments for the five chondrites investigated are listed in Table 1. The data for rhodium and silver are the averages of three and two determinations, respectively, for each meteorite. The relative error for the rhodium analysis does not exceed 30 per cent, the silver results are good by a factor of 2 only. The indium data which are an upper limit only are the results of one run for each meteorite. The counting rate of the indium samples was so low that neither good counting statistics could be achieved nor an unambiguous analysis of the decay curves could be obtained.

Table 1. Abundance of rhodium, silver and indium (p.p.m.)

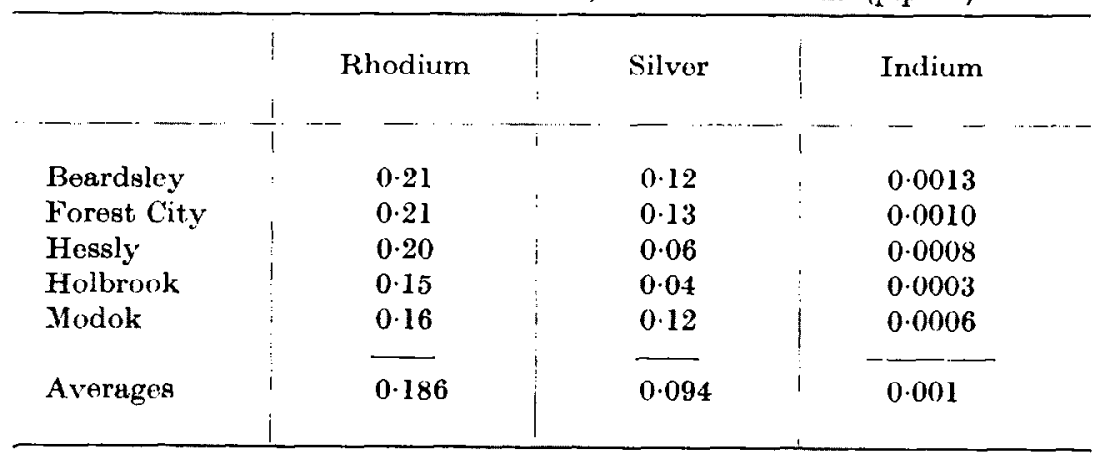

Table 2. Atomic abundance of rhodium, silver and indium relative to silicon $=10^{6}$

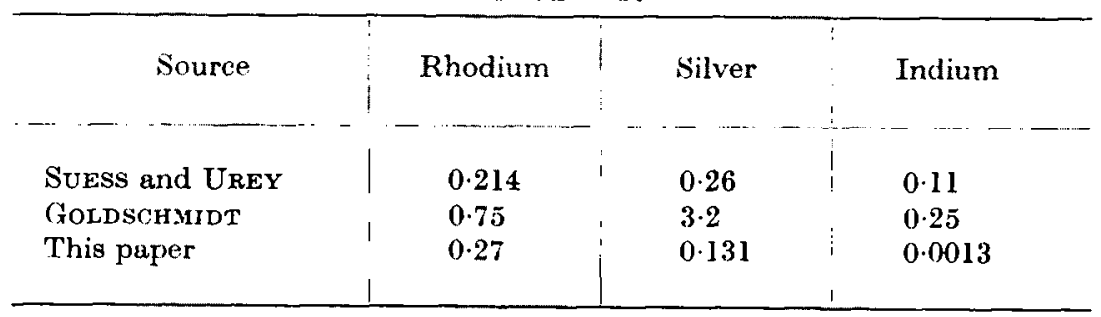

The counting rates obtained in the experiments with Beardsley are: 6000 $\mathrm{c} / \mathrm{min}$ of $4.4 \mathrm{~min} \mathrm{Rh}^{104} 10$ min after the end of the bombardment with a background of long lived contaminations of $350 \mathrm{c} / \mathrm{min}, 3300 \mathrm{c} / \mathrm{min}$ of $2.3 \mathrm{~min} \mathrm{Ag}^{108}$ $5.5 \mathrm{~min}$ after the end of bombardment with a long-lived background of $180 \mathrm{c} / \mathrm{min}$. The constant normal background of the counter was $65 \mathrm{c} / \mathrm{min}$. In all experiments for rhodium and silver the decay curve could be analysed without difficulties with the expected half-lives resulting. 


\section{U. SCHINDEWOLF and M. WAFLGKEN}

From the average data of Table 1 the atomic abundance of the three elements relative to silicon $=10^{6}$ have been calculated and compared with the ones listed by SUESS and UREY (1956) and Goldschuid (1954). The agreement of the new rhodium data and the ones estimated by Suess and UREY is good. Silver seems to be a factor of 2 lower than estimated. (SuEss and UREY state that the results of most trace silver determinations are too high due to contamination of equipment and hands of the experimentors. During the course of the analysis we have avoided any handling of silver coins, tableware, etc. to exclude contamination of this kind.) Indium seems to be lower by a factor of 100 or more than the estimations based on interpolation by Suess and UREY.

The apparent low abundance of indium in the meteorites as compared to the estimations cannot be understood easily. In the activation analysis described for indium only the concentration of the one isotope $\mathrm{In}^{115}$ was measured. The assumption normally can be made that the isotopic composition of matter is very uniform, and even for meteorites (which might come from other solar systems) no exceptions have been observed.

Because $\mathrm{In}^{115}$ has a metastable state $(1 / 2-), 335 \mathrm{keV}$ above ground state which decays by $\beta$-emission with a partial half-life of $100 \mathrm{hr}$ to $\mathrm{Sn}^{15}$, it might be conceivable that via thermal excitation $\mathrm{In}^{115}$ has been depleted. 'This could explain the very low abundance of $\operatorname{In}^{115}$ in meteorites. However, the temperatures necessary for the thermal excitation are of the order of $10^{10}$ degrees and it seems very unlikely that the matter has been at such a temperature for a time long enough to allow the $\mathrm{In}^{115}$ to decay.

Nevertheless, an experiment has been performed to determine the abundance of $\mathrm{In}^{113}$ by activation and counting its neutron capture product 50 day $\operatorname{In}^{114 m}$, which is formed with a capture cross-section of 61 barns. After irradiating $1 \mathrm{~g}$ samples of Modok for a week with a flux of $10^{13}$ neutrons-sec ${ }^{-1}-\mathrm{cm}^{-2}$ together with a standard of $1 \mu \mathrm{g}$ of indium at the Argonne CP5 Reactor and processing them in a manner similar to that described above, here too only an upper limit of 0.001 p.p.m. for indium can be set. This confirms that the isotopic ratio of indium in meteorites is not much different from that on earth, it probably is even the same, and that the abundance of indium in chondrites is very low indeed.

By using spectrographic analysis with a sensitivity of 0.02 p.p.m. SHAw (1952a) also was unable to detect any indium in chondrites. So far there is not any reasonable explanation for the low indium content of the meteorites. Because in nucleogenesis $\mathrm{In}^{115}$ has the same chance to be formed as the other unshielded nuclides with odd mass numbers of this mass area one only can conclude that indium was depleted in the meteorites by some chemical or physical fractionation. The reported low value for thallium (SHAw, 1952b) is also of interest in this connexion.

Acknouledgements - This work was supported by the U.S. Atomic Energy Commission and tho Michigan Memorial Phoenix Project. Thanks are due to the reactor staffs of the Ford Nuclear Reactor at the Cniversity of Michigan and of the CP5 Reactor at Argonne National Laboratory for arranging for the irradiations. The writers are very much obliged to Dr. W. W. MEINke for his help and encouragement. 'They are greatly indebted to Professor E. W. Hainrich, the Curator of Mineralogical Collections, at the University of Michigan for most of the samples 
used. The interest of Professors H. C. UREY and J. H. HuIzengA in this work is also acknowledged. One of us (U. S.) is gratoful to Dr. Meinke for the invitation to work in the Nuclear Chemistry Group of the Chemistry Department of the University of Michigan.

\section{REFERENCES}

Alexandfr J. M., Schindewolf U. and Coryeli. C. 1). (1958) Phys. Rev. 111, 248. Goldschmidt V. M. (1954) Geochemistry. Clarendon Press, Oxford.

Mrinkf W. W. (1959) Rapid activation analysis with the Ford nuclear reactor. Nucleonics $17,86$.

ScHindewolf U. (1958) Angew. Chem. 70, 181.

Shaw 1), M. (1952a) Geochim. et Cosmochim. Acta 2, 118.

Shaw D. M. (1952b) Geochim. et Cosmochim. Acta 2, 185.

Suess H. F. and Urey H. C. (1956) Rev. Mod. Phys. 28, 53.

Sunderman D. N., Ackermany I. B. and Mriske W. W. (1959) Analyt. Chem. 31, 40. 